\title{
Práctica basada en evidencia en estudiantes de enfermería colombianos
}

\section{Competence for evidence based practice among nursing students in an university in cúcuta (Colombia)}

\section{Competências para a prática baseada em evidências em estudantes de enfermagem de uma universidade em cúcuta (Colômbia)}

\author{
María del Pilar Ureña-Molina* \\ Isabel María López-Medina** \\ Pedro Luis Pancorbo-Hidalgo ${ }^{* * *}$
}

\section{Para citar este artículo / To reference this article / Para citar este artigo/}

Ureña-Molina MP, Lopez-Medina IM, Pancorbo-Hidalgo PL. Práctica basada en evidencia en estudiantes de enfermería colombianos. Rev. cienc. cuidad. 2017; 14(2):51-64.

\section{Resumen}

Objetivo. Determinar el nivel de competencias sobre Práctica Basada en Evidencia en estudiantes de un programa de Enfermería de Colombia. Materiales y métodos. Mediante un diseño cuantitativo, descriptivo y correlacional, el estudio se desarrolló en 2 fases. La primera fase consistió en la adaptación cultural y validación de contenido del cuestionario de Competencias en Práctica Basada en la Evidencia (CACH-PBE) al contexto Colombiano; este instrumento valora tres factores: Actitudes, Habilidades y Conocimientos. En la segunda fase se aplicó el cuestionario a una muestra de 291 estudiantes del Programa de Enfermería de una Universidad de Cúcuta, Colombia, durante el primer semestre del 2016. Resultados. Se encontró un promedio de edad de 20 años, de género femenino en su mayoría, el 57,4 \% de ellos no tenía ninguna formación previa sobre PBE y el 57.1 \% la había recibido en metodología de investigación. La puntuación global de competencia en PBE fue de 3,58 sobre un máximo de 5, destacando la dimensión Actitudes hacia la PBE con mayor puntuación y la dimensión Conocimientos con menor; encontrándose diferencias estadísticamente significativas a mayor medida en que los estudiantes se encontraban en un semestre superior. Conclusiones. Los estudiantes obtuvieron un nivel medio en competencias para la PBE, asociándose un impacto positivo de la formación académica recibida, ya que en los últimos semestres se evidenció mayor puntuación con respecto a los primeros.

Palabra clave: Enfermería basada en evidencia, conocimiento, aptitud, estudiantes de enfermería.

\section{Abstract}

Objective: To determine the level of competence regarding Practice Based on Evidence in students of a nursing program in Colombia. Materials and methods: Through a quantitative, descriptive, and correlational design, the study was developed in two phases. The first phase, consisted on the cultural adaptation and validation of the content of the questionnaire of Competitions in Practice Based on Evidence (CACH-PBE) to the Colombian context; this instrument evaluates 3 factors: attitudes, skills, and understanding. In the second phase, the questionnaire was applied to a sample of 291 students of the nursing program of a university in Cucuta- Colombia during the first semester of 2016. Results: An average age of 20 years was found, mostly females, $57.4 \%$ did not have previous training about PBE and $57.1 \%$ had received training of PBE in methodology of research. The global score in PBE competence was of 3.58 out of a maximum of 5; highlighting the Attitude dimension toward the PBE with the highest score and the Knowledge dimension with the lowest

ISSN-PRINT

$1794-9831$

E-ISSN 2322-7028

Vol. 14 No. 2

Jul - Dic 2017

Cúcuta, Colombia

Recibido:

31 de octubre de 2016

Aprobado:

17 Mayo de 2017

* Enfermera

Magister en

Enfermería.

Correo electrónico: mariadelpum@ ufps.edu.co. Orcid: 0000-0003-21843595. Docente.

Universidad Francisco de Paula Santander. Cúcuta, Colombia.

** Enfermera.

Doctora en

Enfermería. Correo: imlopez@ ujaen.es. Orcid: 0000-0002-34379229. Profesora Universidad de Jaén. Jaén, España. *** Diplomado en Enfermería. Doctor en Biología. Licenciado en Ciencias Biológicas. Correo: pancorbo@ ujaen.es. Orcid: 0000-0001-81578004. Profesor, Universidad de Jaén. Jaén, España. 
ISSN-PRINT

1794-9831

E-ISSN 2322-7028

Vol. 14 No. 2

Jul - Dic 2017

Cúcuta, Colombia

score; encountering statistically significant differences to a greater extent as the students moved ahead to a higher semester. Conclusions: The students received an average level in competences for the PBE, associating a positive impact with the academic training received, considering that in the last semesters a higher score was evidenced compared to the first ones.

Keywords: Nursing based on evidence, knowledge, attitude, nursing students.

\section{Resumo}

Objetivo. Determinar o nível de competências sobre Prática Baseada na Evidencia (PBE) de estudantes de um curso de Enfermagem na Colômbia. Materiais e métodos. O estudo se desenvolveu em duas fases, através um delineamento quantitativo, descritivo e correlacional, A primeira fase, consistiu na adaptação cultural e validação de conteúdo do questionário de Competências em Prática Baseada na Evidencia (CACH-PBE) no contexto Colombiano; este instrumento valora três fatores: Atitudes, Habilidades e Conhecimentos. Na segunda fase, aplicou-se o questionário a uma amostra de 291 estudantes do Programa de Enfermagem de uma Universidade localizada na cidade de Cúcuta - Colômbia, durante o primeiro semestre de 2016. Resultados. Encontrou-se uma média de idade de 20 anos, de género feminino em sua maioria, o 57,4\% deles não tinham nenhuma formação previa sobre PBE e o $57,1 \%$ a receberam em metodologia da pesquisa. A pontuação global de competência em PBE foi de 3,58 sobre um máximo de 5; destacando a dimensão Atitudes para a PBE com maior pontuação e a dimensão Conhecimentos com menor; encontrando-se diferenças estatisticamente significativas a maior medida em que os estudantes se encontravam num semestre superior. Conclusões. Os estudantes obtiveram um nível médio em competências para a PBE, associando-se um impacto positivo da formação académica recebida, já que nos últimos semestres se evidenciou maior pontuação respeito dos primeiros.

Palavras-chave: Enfermagem baseada na evidencia, conhecimento, atitude, estudantes de enfermagem.

\section{Introducción}

De manera histórica, la Enfermería Basada en la Evidencia (EBE) tiene como base el modelo de la Medicina Basada en la Evidencia (MBE) y los aportes realizados por Florence Nightingale (1820-1910) al considerar la utilización de los resultados estadísticos para la mejora en la atención de los pacientes. Algunos autores como Muhdall (1) e Ingersoll (2) la definen como la incorporación de la información derivada de la teoría y la investigación para la toma de decisiones en la prestación de los cuidados, teniendo en cuenta sus preferencias y necesidades individuales.

En la actualidad es reconocido que una práctica de cuidado basada en la evidencia resulta necesaria para proporcionar cuidados de la salud en forma equitativa y efectiva para toda la población, favorece la mejor costo/efectividad de los recursos y establece la mejor condición posible para ofrecer un excelente cuidado a los pacientes $(3,4)$.

Sin embargo, al revisar la implementación de la práctica basada en la evidencia por parte de los profesionales de enfermería, se han identificado algunas barreras que obstaculizan su total desarrollo, tales como la falta de reconocimiento de la enfermería como una profesión autónoma, la falta de incentivos para dedicarse a la investigación o el déficit de formación metodológica, la limitada disponibilidad y baja utilización de la evidencia en enfermería, la falta de comunicación entre la academia y los entornos de práctica clínica, la carga de trabajo, la falta de apoyo de la administración y una cultura de trabajo resistente al cambio, entre otras (5-11), situación que tampoco es ajena a otras disciplinas del área de la salud, donde igualmente se presentan obstáculos para la implementación de la Práctica Basada en la Evidencia (PBE) (12).

A nivel mundial, instituciones como el Johanna Briggs Institute (JBI) y el Centre for Evidence-Based Nursing en Reino Unido, han sido fundamentales en el movimiento de la EBE, dedicadas a proporcionar guías de práctica clínica de calidad que reflejan la necesidad de basar la enfermería en las creencias, 
valores y el conocimiento en los que se apoya la práctica, así como en la mejor evidencia existente (8).

De acuerdo con Eterovic y Stiepovich (2) "la comunidad académica debe asumir el compromiso de empoderar al estudiante en el ejercicio de una práctica futura en que se considere y valore el contexto cultural de la práctica, el reconocimiento del individuo como ser holístico, la reflexión e indagación como forma de vida en el quehacer cotidiano, las habilidades de búsqueda de información, la exploración sistemática de resolución de problemas y la publicación de los hallazgos de su investigación", aspectos que son soportados con la rigurosidad de la investigación científica al estudiar el conocimiento en torno al cuidado de la salud desde un abordaje biomédico u objetivo y de la comprensión individualizada de cada experiencia de cuidado, a través de los enfoques metodológicos cuantitativo y cualitativo, que resultan en una herramienta poderosa para el estudio y análisis de la práctica de Enfermería y su transformación $(13,14)$.

Son escasos los estudios realizados en estudiantes de enfermería que demuestran el nivel alcanzado de formación en práctica basada en la evidencia (15). Se han desarrollado algunos instrumentos específicos para medir este concepto (16-19) y la eficacia de los cursos de PBE (20). Recientemente, en una universidad de España, se desarrolló una investigación con estudiantes de enfermería de todos los cursos de un programa académico donde se incluyó una asignatura específica de PBE, cuyos resultados evidenciaron un adecuado nivel de competencia en PBE, ocurriendo el mayor incremento el último curso de la carrera (21).

Demanerasimilar,enEcuador, un estudioexploró acerca de las creencias, las oportunidades de implementación y el apoyo que una institución universitaria da a la formación de enfermería basada en evidencia (EBE) desde la perspectiva de docentes y estudiantes, teniendo en cuenta los cinco pasos del método EBE y otros criterios. Sus hallazgos evidenciaron una alta valoración sobre creencias referidas a la EBE y una baja implementación de cambios en la práctica o enseñanza bajo este enfoque (22).

En el contexto colombiano, un estudio realizado en cuatro programas de pregrado de enfermería de
Santander (23) demostró "que la capacidad de analizar críticamente sobresale como una de las competencias que se fomenta en el estudiante cuando se utiliza la enfermería basada en la evidencia"; sin embargo, se resaltó que las competencias "de los estudiantes de enfermería para enfrentar el ejercicio de su profesión, aún no se ven sustentadas en las ventajas que puede desarrollar el uso de la enfermería basada en la evidencia, como estrategia" para el cuidado de los pacientes (23).

Dada la relevancia que cobra la formación en investigación en los profesionales de enfermería para su producción o aplicación, es primordial que durante su proceso de formación de pregrado el estudiante adquiera las competencias y habilidades necesarias para la implementación de una práctica basada en la evidencia que le permita sustentar su accionar con un fundamento científico y pensamiento crítico, en aras de retroalimentar los cuidados impartidos y validar la toma de decisiones en cualquier entorno de cuidado.

El presente artículo muestra los resultados de un estudio que se realizó con el objetivo de determinar el nivel de competencia en práctica basada en evidencia en estudiantes de un programa de enfermería de Colombia, partiendo del escaso conocimiento sobre esta temática en el contexto local y nacional, para que, sobre la base de estos hallazgos, se deriven estrategias tendientes a fortalecer y empoderar la formación de EBE en la comunidad académica y científica.

\section{Materiales y métodos}

Para esta investigación se usó un diseño cuantitativo, descriptivo y correlacional. Se desarrolló en 2 fases: en la primera se hizo la adaptación cultural a Colombia y la validación de contenido del cuestionario, y en la segunda fase se hizo la recogida de datos en la muestra de estudiantes de enfermería.

En este estudio se usó el Cuestionario de Competencias en Práctica Basada en la Evidencia (CACH-PBE), en versión española, de Ruzafa et al. (17), proporcionado por su autora junto con el permiso de uso y adaptación cultural, el cual consta de 25 ítems agrupados en 3 factores: actitud ante la práctica basada en la evidencia, habilidades para la PBE y conocimientos sobre la PBE. En su versión original presentó buenos valores de fiabilidad (consistencia interna alfa Cronbach $=0,88$ ) y validez de constructo y discriminante (17). 
ISSN-PRINT

1794-9831

E-ISSN 2322-7028

Vol. 14 No. 2

Jul - Dic 2017

Cúcuta, Colombia
Con el fin de asegurar su adaptación cultural y lingüística al entorno de Colombia, el cuestionario fue revisado por 3 docentes de la facultad de enfermería de una Universidad en Colombia, quienes propusieron algunos cambios en palabras o redacción de algunos ítems.

Esta nueva versión del cuestionario CACH-PBE se sometió a validación de contenido por un panel de 8 expertos académicos procedentes de diversas facultades de enfermería de universidades de Colombia. Para cada ítem se valoró su relevancia y su claridad, puntuando entre 1 a 5, según menor a mayor grado. Para establecer el grado de acuerdo entre los expertos se utilizó el índice V de Aiken, media y su intervalo de confianza del $95 \%(24,25)$. Para la relevancia, en el factor Actitud se identificaron 3 ítems con menor grado de acuerdo (límite inferior del IC $95 \%$ del índice V Aiken <0,50). En los factores Habilidades y Conocimientos, ningún ítem puntuó menos de 0,50. Para la claridad, el número de ítems con menos acuerdo (límite inferior del IC $95 \%<$ 0,50) fueron: Actitud, 4 ítems; Habilidades, 1 ítem; Conocimientos, 4 ítems. En estos ítems se revisó su redacción y, en caso necesario, se hizo algún ajuste en la misma. Se obtuvo una versión final del cuestionario CACH-PBE adaptada culturalmente a Colombia.

La población de estudio fueron los estudiantes del programa de enfermería de una universidad pública en Cúcuta, Colombia. Los estudiantes matriculados en el I semestre del año 2016 fueron 318, contando con una participación en total de 291 estudiantes en el desarrollo del estudio. Este programa posee 8 semestres de formación académica, cada uno de los cuales tiene una duración de 16 semanas del calendario, correspondiendo a un total de 4 años. La formación en el área de investigación es uno de los ejes transversales en la formación del estudiante y se inicia desde los primeros semestres con cursos específicos para que el estudiante desarrolle las competencias básicas y elementales para la dirección de proyectos de investigación en las diferentes áreas del cuidado de enfermería que se ejecutan durante la carrera.

La variable principal (variable dependiente) fue la competencia para la PBE y los 3 factores que la forman: actitud, habilidades y conocimientos. Se midió mediante el cuestionario CACH-PBE en versión en español adaptada a Colombia. La escala de respuesta de los ítems es de tipo Likert de 5 puntos (1 Muy en desacuerdo a 5 Muy de acuerdo).
Como variables independientes se tuvieron: variables demográficas y formativas de los estudiantes, valoración autodeclarada sobre actitudes hacia la PBE y conocimientos metodológicos, medidas en una escala entre 0 y 10 puntos. Para los estudiantes de los semestres $1^{\circ}$ a $7^{\circ}$ se administraron los cuestionarios en papel, para ser autocumplimentados. Los estudiantes de $8^{\circ}$ semestre completaron los cuestionarios on-line (mediante SurveyMonkey).

Las hipótesis a testar fueron:

- La puntuación en competencias en PBE será mayor en los estudiantes que cursan un semestre académico superior.

- La puntuación en competencias en PBE será mayor en los estudiantes con más horas de formación en metodologías de investigación y PBE.

- No habrá diferencias en la puntuación en competencias en $\mathrm{PBE}$ según el género de los estudiantes.

Los datos fueron tabulados y codificados para realizar el análisis estadístico. De acuerdo con las instrucciones de puntuación del cuestionario $\mathrm{CACH}$ PBE, se invirtió la puntuación de varios de los ítems, se calculó la puntuación global en el cuestionario y la puntuación parcial en cada uno de los 3 factores, en un rango entre 1 y 5 .

Para el análisis descriptivo se usaron frecuencias y porcentajes para las variables categóricas; y medias y desviación estándar, o bien mediana y amplitud intercuartil (según tipo de distribución), para las variables ordinales y cuantitativas. El ajuste de las variables a la distribución normal se evaluó mediante el test de Kolmogorov-Smirnoff. Las variables puntuación global, actitud, habilidades y conocimientos del cuestionario CACH-PBE se ajustaron a la distribución normal; el resto de variables no tenía distribución normal.

Para las correlaciones se empleó el test de Spearman o test $\mathrm{R}$ de Pearson. Las hipótesis de igualdad de medias en las puntuaciones del cuestionario CACH-PBE se testaron mediante la prueba $t$ de Student, para la asociación con el género; o ANOVA, para la asociación con curso académico y horas de formación (debido a que en la mayoría de casos no se cumplía el supuesto de igualdad en las varianzas, se usó la prueba robusta 
de Welch y el estadístico T2 Tamhane para pruebas post hoc). El tamaño de efecto se midió mediante la diferencia de medias y su intervalo de confianza del 95 $\%$. Se usaron test de 2 colas, considerando un valor de $\mathrm{p}<0,05$ con significación estadística. Para el análisis se usó el programa SPSS Versión $\mathrm{N}^{\circ} 15$.

Los datos obtenidos se agruparon cada dos semestres en un año. La distribución por años fue: $28,2 \%(n=82)$ de primer año, 23,7 \% $(\mathrm{n}=69)$ de segundo año, 26,1 $\%(n=76)$ de tercer año y $22 \%(n=64)$ de cuarto año.

El estudio se adhirió a las normas éticas para la investigación con seres humanos, al garantizar la privacidad y no divulgación de los resultados con nombre propio de los estudiantes; así como también la protección de los valores éticos como la autonomía, no maleficencia, beneficencia, justicia y confidencialidad, considerándose un estudio de bajo riesgo para la salud (26). En este sentido, se obtuvo la autorización de los responsables académicos de la facultad y los estudiantes firmaron su consentimiento para completar los cuestionarios, garantizando el anonimato y la confidencialidad en los datos recogidos.

\section{Objetivos}

\section{Objetivo general}

Determinar el nivel de competencia para la Práctica de Enfermería Basada en Evidencia (PBE) en estudiantes de Enfermería de la Universidad Francisco de Paula Santander.

\section{Objetivos específicos}

- Determinar las características sociodemográficas y formativas de los estudiantes de enfermería.

- Determinar el nivel de actitudes, habilidades y grado de conocimiento sobre la PBE en los estudiantes de enfermería.

- Establecer la existencia de asociación entre la puntuación en el nivel de competencia en PBE y factores demográficos y formativos de los estudiantes.

- Establecer el nivel autovalorado por los estudiantes sobre varios aspectos asociados a la PBE.

El período de recolección de la información se realizó en los meses de febrero a junio del año 2016. Como limitaciones se encontró un porcentaje de respuesta cercano al $9 \%$ de la población de estudiantes que no diligenciaron el cuestionario.

\section{Resultados}

\section{Características sociodemográficas y formativas de los estudiantes de enfermería.}

Para la variable edad, el valor de la mediana fue de 20 años $\left(\mathrm{P}_{25}: 19-\mathrm{P}_{75}: 21\right)$ y en relación con el género, predominantemente eran mujeres, con un $77 \%$ $(\mathrm{n}=224)$. Un $86 \%$ del alumnado $(\mathrm{n}=252)$ no poseía otro título académico previo y un 83,5\% $(\mathrm{n}=243)$ no desempeñaba ningún trabajo remunerado en esos momentos. Con respecto a las características formativas de la muestra, un 57,4 \% ( $=167)$ no tenía ninguna formación previa sobre PBE y sólo un $24 \%(\mathrm{n}=72)$ había recibido formación sobre el tema con una duración inferior a $40 \mathrm{~h}$. Para la formación en metodología de investigación, un 42,6\% $(\mathrm{n}=124)$ carecía de formación alguna, un 26,5 \% ( $\mathrm{n}=77)$ había recibido entre 40 y $150 \mathrm{~h}$ de formación y apenas en un $6,9 \%(n=20)$ su formación superaba las $150 \mathrm{~h}$. En la Tabla 1 se detallan las características sociodemográficas y formativas de los sujetos de estudio.

\section{Competencias para PBE en estudiantes de enfermería.}

E-ISSN 2322-7028

Vol. 14 No. 2

Jul - Dic 2017

Cúcuta, Colombia 
ISSN-PRINT

1794-9831

E-ISSN 2322-7028

Vol. 14 No. 2

Jul - Dic 2017

Cúcuta, Colombia

Tabla 1. Características sociodemográficas y formativas del alumnado de Enfermería.

\begin{tabular}{ll}
\hline Variables & Frecuencia (\%) \\
\hline Edad (años): (mediana; $\left.\mathrm{P}_{25}-\mathrm{P}_{75}\right)$ & $20 ; 19-21$ \\
\hline Género: $\mathrm{n}(\%)$ & \\
Mujer & $224(77 \%)$ \\
Hombre & $67(23 \%)$ \\
\hline Otros estudios previos al Grado: $\mathrm{n}(\%)$ & \\
No & $252(86 \%)$ \\
Sí & $38(13,1 \%)$ \\
$\quad$ Técnicos / ciclos formativos & $1(0,3 \%)$ \\
$\quad$ No consta & $37(12,7 \%)$ \\
Datos perdidos & $1(0,3 \%)$ \\
\hline Formación PBE: $\mathrm{n}(\%)$ & \\
Ninguna & $167(57,4 \%)$ \\
$<40 \mathrm{~h}$ & $72(24,7 \%)$ \\
$40-150 \mathrm{~h}$ & $42(14,4 \%)$ \\
$>150 \mathrm{~h}$ & $8(2,7 \%)$ \\
Datos perdidos & $2(0,7 \%)$ \\
\hline Formación metodología investigación: $\mathrm{n}(\%)$ & \\
Ninguna & $124(42,6 \%)$ \\
$<40 \mathrm{~h}$ & $69(23,7 \%)$ \\
$40-150 \mathrm{~h}$ & $77(26,5 \%)$ \\
$>150 \mathrm{~h}$ & $20(6,9 \%)$ \\
Datos perdidos & $1(0,3 \%)$ \\
\hline Trabajo actual remunerado: $\mathrm{n}(\%)$ & \\
No & $243(83,5 \%)$ \\
Sí & $48(16,5 \%)$ \\
\hline &
\end{tabular}

Fuente: Cuestionario de Competencias en Práctica Basada en la Evidencia (CACH-PBE), 2014.

En la Tabla 2 se muestran las puntuaciones globales del cuestionario de PBE y de sus dimensiones -actitud, habilidades y conocimientos- desglosadas según el año al que pertenece el alumnado estudiado. En todo el conjunto, la puntuación global en competencia en PBE fue de 3,58 sobre un máximo de 5, destacando la dimensión Actitud hacia la PBE con mayor puntuación y la dimensión conocimientos con menor. Tanto para la puntuación global como para las sub-escalas del cuestionario se detectó que, en términos generales, ésta es mayor a medida que los estudiantes se encuentran en semestres superiores. Se obtuvieron diferencias significativas de la puntuación global y las subescalas por año, confirmando la hipótesis propuesta (puntuación global: Welch 42,96, p $<0,001$; actitud: Welch 25,12, $\mathrm{p}<0,001$; habilidades: Welch 50,17, $\mathrm{p}<0,001 ;$ y conocimientos: Welch 21,01, $\mathrm{p}<0,001$ ). El análisis de las correlaciones entre la puntuación global de PBE y sus dimensiones mostró que la puntuación global en PBE correlaciona bien con cada una de las tres dimensiones, siendo más fuerte para las Actitudes ( $\mathrm{r}$ Pearson $=0,85, \mathrm{p}<0,001$ ), que para las Habilidades ( $\mathrm{r}$ Pearson $=0,77, \mathrm{p}<0,001$ ) o Conocimientos ( $r$ Pearson $=0,51, p<0,001)$.

En el análisis de correlaciones internas entre las dimensiones resultaron correlaciones estadísticamente significativas entre las habilidades y las actitudes ( $\mathrm{r}$ Pearson $=0,50, p<0,001)$ y entre las habilidades y los conocimientos ( $\mathrm{r}$ Pearson $=0,32, \mathrm{p}<0,001)$, si bien las actitudes y los conocimientos no correlacionaron de forma adecuada ( $\mathrm{r}$ Pearson $=0,06, \mathrm{p}=0,33$ ). 
Tabla 2. Puntuación global en PBE y en las sub-escalas de actitud, habilidades y conocimientos del alumnado de Enfermería.

\begin{tabular}{lllllllllll}
\hline Años* & $\mathrm{n}$ & \multicolumn{2}{c}{ Puntuación Global PBE } & \multicolumn{2}{c}{ Actitud hacia PBE } & \multicolumn{2}{c}{ Habilidades para PBE } & \multicolumn{2}{c}{ Conocimientos sobre PBE } \\
& & Media (IC95\%) & d.e. & Media (IC95\%) & d.e. & Media (IC95\%) & d.e. & Media (IC95\%) & d.e \\
\hline $1^{\circ}$ & 82 & $3,41(3,34-3,48)$ & 0,31 & $3,73(3,62-3,83)$ & 0,49 & $3,38(3,28-3,49)$ & 0,48 & $2,74(2,60-2,88)$ & 0,64 \\
\hline $2^{\circ}$ & 69 & $3,30(3,24-3,37)$ & 0,26 & $3,48(3,38-3,58)$ & 0,42 & $3,02(2,95-3,08)$ & 0,25 & $3,21(3,15-3,27)$ & 0,27 \\
\hline $3^{\circ}$ & 76 & $3,73(3,65-3,82)$ & 0,35 & $3,94(3,83-4,04)$ & 0,46 & $3,70(3,57-3,81)$ & 0,53 & $3,34(3,20-3,48)$ & 0,62 \\
\hline $4^{\circ}$ & 64 & $3,89(3,78-3,40)$ & 0,43 & $4,15(4,02-4,28)$ & 0,52 & $3,71(3,56-3,86)$ & 0,60 & $3,51(3,37-3,66)$ & 0,58 \\
\hline \multirow{2}{*}{ TOTAL } & $\mathbf{2 9 1}$ & $\mathbf{3 , 5 8 ( 3 , 5 3 - 3 , 6 2 )}$ & $\mathbf{0 , 4 1}$ & $\mathbf{3 , 8 2 ( 3 , 7 6 - 3 , 8 8 )}$ & $\mathbf{0 , 5 3}$ & $\mathbf{3 , 4 5 ( 3 , 3 8 - 3 , 5 1 )}$ & $\mathbf{0 , 5 6}$ & $\mathbf{3 , 1 8 ( 3 , 1 0 - 3 , 2 5 )}$ & $\mathbf{0 , 6 2}$ \\
\hline
\end{tabular}

IC95\%: Intervalo de confianza al 95\%; d.e.: desviación estándar.

* Cada año incluye 2 semestres

Fuente: Cuestionario de Competencias en Práctica Basada en la Evidencia (CACH-PBE), 2014.

Al realizar los contrastes de diferencias de medias de puntuaciones por años, se estableció que dichas diferencias son significativas $(p<0,05)$ entre las puntuaciones de cuarto respecto a las de primer y segundo año, tanto para la puntuación global como para las actitudes, las habilidades y los conocimientos sobre PBE; si bien estas diferencias no fueron significativas entre cuarto y tercer año para ninguna dimensión (ver Tabla 3).

También se obtuvieron diferencias de medias positivas significativas entre la puntuación global y las tres dimensiones del tercer año con respecto al primer año; si bien al comparar tercero con segundo, sus diferencias fueron significativas sólo para la puntuación global, las actitudes y las habilidades. Sin embargo, hay que destacar que las puntuaciones de los estudiantes de segundo año, tanto global como en sus tres dimensiones, fue menor que las del alumnado de primer año, siendo además estas diferencias estadísticamente significativas para cada una de las dimensiones aisladas, aunque no para la puntuación global del cuestionario.

Al comparar las dimensiones actitud, habilidades y conocimientos sobre PBE, así como la puntuación global, con la formación previa que había recibido el alumnado sobre PBE, se obtuvieron diferencias estadísticamente significativas en las puntuaciones de habilidades para PBE (test ANOVA; Welch: 8,77, $\mathrm{p}<0,001$ ) y de conocimientos sobre PBE (Welch: $9,50, p<0,001)$, encontrando una mayor puntuación de conocimientos en los que reciben 40 o más horas de formación en relación con los que no reciben ninguna formación de $\operatorname{PBE}(\mathrm{p}<0,05)$.

Con respecto a la formación previa en metodología de investigación, de nuevo se obtuvieron diferencias estadísticamente significativas con respecto a las habilidades para PBE (Welch: $3,63, p=0,02$ ) y a los conocimientos (Welch: 13,01, p<0,001) y, además, en esta ocasión, para la puntuación global en PBE (Welch: 5,63, p=0,002). Concretamente, tanto la puntuación global como la obtenida en habilidades y en conocimientos aumentan a mayor número de horas de formación en metodología de investigación, si bien dichas diferencias sólo son significativas $(p<0,05)$ en el grupo que ha recibido una formación de 40 a $150 \mathrm{~h}$.
ISSN-PRINT

1794-9831

E-ISSN 2322-7028

Vol. 14 No. 2

Jul - Dic 2017

Cúcuta, Colombia 
ISSN-PRINT

1794-9831

E-ISSN 2322-7028

Vol. 14 No. 2

Jul - Dic 2017

Cúcuta, Colombia

Tabla 3. Diferencias de puntuación global, actitud, habilidades y conocimientos por años académicos.

\begin{tabular}{|c|c|c|c|c|c|c|}
\hline \multirow{2}{*}{$\begin{array}{l}\text { Contrastes por } \\
\text { años }\end{array}$} & \multicolumn{3}{|c|}{ Actitud hacia PBE } & \multicolumn{3}{|c|}{ Habilidades para PBE } \\
\hline & DM & IC95\% & $\mathrm{p}$ & DM & IC95\% & $\mathrm{p}$ \\
\hline \multicolumn{7}{|l|}{ Cuarto } \\
\hline Primero & 0,42 & 0,19 a 0,64 & $<0,001$ & 0,32 & 0,77 a 0,57 & 0,004 \\
\hline Segundo & 0,66 & 0,44 a 0,89 & $<0,001$ & 0,69 & 0,47 a 0,91 & $<0,001$ \\
\hline Tercero & 0,21 & $-0,18$ a 0,43 & 0,08 & 0,02 & - 0,24 a 0,28 & 1,00 \\
\hline \multicolumn{7}{|l|}{ Tercero } \\
\hline Primero & 0,21 & 0,01 a 0,41 & 0,04 & 0,31 & 0,09 a 0,52 & 0,001 \\
\hline Segundo & 0,46 & 0,26 a 0,65 & $<0,001$ & 0,67 & 0,49 a 0,86 & $<0,001$ \\
\hline \multicolumn{7}{|l|}{ Segundo } \\
\hline Primero & $-0,25$ & $-0,44$ a $-0,05$ & 0,006 & $-0,37$ & $-0,53$ a $-0,20$ & $<0,001$ \\
\hline \multirow[t]{2}{*}{$\begin{array}{c}\text { Contrastes por } \\
\text { años }\end{array}$} & \multicolumn{3}{|c|}{ Conocimientos sobre PBE } & \multicolumn{3}{|c|}{ Puntuación Global PBE } \\
\hline & $\mathrm{DM}$ & IC95\% & $\mathrm{p}$ & $\mathrm{DM}$ & IC95\% & $\mathrm{p}$ \\
\hline \multicolumn{7}{|l|}{ Cuarto } \\
\hline Primero & 0,77 & 0,50 a 1,04 & $<0,001$ & 0,48 & 0,31 a 0,65 & $<0,001$ \\
\hline Segundo & 0,30 & 0,09 a 0,52 & 0,002 & 0,58 & 0,42 a 0,75 & $<0,001$ \\
\hline Tercero & 0,17 & $-0,10$ a 0,45 & 0,425 & 0,15 & $-0,03$ a 0,33 & 0,135 \\
\hline \multicolumn{7}{|l|}{ Tercero } \\
\hline Primero & 0,60 & 0,32 a 0,86 & $<0,001$ & 0,32 & 0,18 a 0,47 & $<0,001$ \\
\hline Segundo & 0,13 & $-0,08$ a 0,34 & 0,49 & 0,43 & 0,29 a 0,57 & $<0,001$ \\
\hline \multicolumn{7}{|l|}{ Segundo } \\
\hline Primero & 0,47 & 0,26 a 0,68 & $<0,001$ & $-0,10$ & $-0,23$ a 0,02 & 0,143 \\
\hline
\end{tabular}

Test ANOVA. DM: Diferencia de medias; IC95\%: Intervalo de confianza al 95\%; $p$ : nivel de significación estadística.

Fuente: Cuestionario de Competencias en Práctica Basada en la Evidencia (CACH-PBE), 2014.

La Tabla 4 muestra los resultados del análisis de las puntuaciones en el cuestionario CACH-PBE en función del género del alumnado. En contra de lo previsto en la hipótesis de partida, se encontraron diferencias significativas en las puntuaciones en la dimensión habilidades y en la competencia global, que fueron mayores en los hombres. En relación con la edad del alumnado estudiado, solamente se encontró correlación positiva con la dimensión conocimientos sobre PBE (Rho Spearman: 0,30, $<<0,001$ ), pero no en las otras dimensiones ni en la puntuación global. 
Tabla 4. Diferencias de puntuación global, actitud, habilidades y conocimientos por género del alumnado de enfermería.

\begin{tabular}{|c|c|c|c|c|c|c|c|}
\hline \multirow[b]{2}{*}{ Género } & \multirow[b]{2}{*}{$\mathrm{n}$} & \multicolumn{3}{|c|}{ Actitud hacia PBE } & \multicolumn{3}{|c|}{ Habilidades para PBE } \\
\hline & & Media (d.e.) & $\mathrm{p}$ & DM (IC95\%) & Media (d.e.) & $\mathrm{p}$ & DM (IC95\%) \\
\hline Mujer & 224 & $3,79(0,52)$ & \multirow{2}{*}{0,06} & \multirow{2}{*}{$\begin{array}{l}-0,13 \\
(-0,28 \text { a } 0,01)\end{array}$} & $3,40(0,55)$ & \multirow{2}{*}{0,004} & $-0,22$ \\
\hline \multirow[t]{2}{*}{ Hombre } & 67 & $3,92(0,54)$ & & & $3,62(0,53)$ & & $(-0,38$ a $-0,07)$ \\
\hline & & \multicolumn{3}{|c|}{ Conocimientos sobre PBE } & \multicolumn{3}{|c|}{ Puntuación Global PBE } \\
\hline Género & $\mathrm{n}$ & Media (d.e.) & $\mathrm{p}$ & DM (IC95\%) & Media (d.e.) & $\mathrm{p}$ & DM (IC95\%) \\
\hline Mujer & 224 & $3,17(0,62)$ & \multirow{2}{*}{0,70} & $-0,03$ & $3,54(0,40)$ & \multirow{2}{*}{0,02} & $-0,13$ \\
\hline Hombre & 67 & $3,20(0,66)$ & & $(-0,20$ a 0,14$)$ & $3,68(0,42)$ & & $(-0,24 a-0,02)$ \\
\hline
\end{tabular}

Test $T$ Student. d.e.: desviación estándar; p: nivel de significación estadística (prueba t-Student); DM: Diferencia de medias; IC $95 \%$ : Intervalo de confianza al $95 \%$

Fuente: Cuestionario de Competencias en Práctica Basada en la Evidencia (CACH-PBE), 2014.

En relación con los aspectos medidos en los estudiantes mediante las 8 escalas de autovaloración, los resultados expresados como mediana (amplitud intercuartil) fueron: actitud hacia PBE: 7,0 $(3,0)$, conocimientos sobre PBE: $6,0(2,0)$, habilidades para PBE: $6,0(2,0)$, actitud propia hacia la promoción de la PBE: $6,0(2,0)$, actitud percibida de los compañeros hacia PBE: 5,0 $(3,0)$, nivel de conocimientos de inglés: $5,0(3,0)$, nivel de conocimientos de informática: 7,0 $(3,0)$, nivel de conocimientos en bioestadística: 5,00 $(3,0)$.

Las correlaciones entre el cuestionario CACH-PBE y las escalas de autovaloración se detallan en la Tabla 5. Se puede apreciar que la puntuación global del cuestionario correlaciona más fuerte con el nivel de conocimientos autodeclarado en bioestadística y en informática (Rho Spearman: 0,40), seguidos de la actitud percibida hacia la $\operatorname{PBE}(\mathrm{Rho}=0,37)$ y de la actitud hacia la promoción de la $\mathrm{PBE}(\mathrm{Rho}=$ 0,36 ), siendo dichas correlaciones estadísticamente significativas $(\mathrm{p}<0,001)$. Para las dimensiones del cuestionario, las correlaciones más fuertes son las de la actitudes hacia la PBE con la actitud percibida ( $R h o=0,37, p<0,001)$, la dimensión habilidades para $\mathrm{PBE}$ con el nivel de conocimientos en informática $(\mathrm{Rho}=0,42, \mathrm{p}<0,001)$ y la dimensión conocimientos sobre PBE con los conocimientos autovalorados $(\mathrm{Rho}=0,44, \mathrm{p}<0,001)$.
ISSN-PRINT

1794-9831

E-ISSN 2322-7028

Vol. 14 No. 2

Jul - Dic 2017

Cúcuta, Colombia 
ISSN-PRINT $1794-9831$

E-ISSN 2322-7028

Vol. 14 No. 2

Jul - Dic 2017

Cúcuta, Colombia

Tabla 5. Correlaciones entre el cuestionario CACH-PBE y las escalas de autovaloración.

\begin{tabular}{lcccc}
\hline & \multicolumn{2}{c}{ Actitud hacia PBE } & \multicolumn{2}{c}{ Habilidades para PBE } \\
\hline Aspectos autovalorados & $\mathrm{R}$ & $\mathrm{p}$ & $\mathrm{R}$ & $\mathrm{p}$ \\
\hline Actitud percibida hacia PBE & 0,37 & $<0,001$ & 0,26 & $<0,001$ \\
\hline Conocimientos autovalorados sobre PBE & 0,08 & 0,15 & 0,16 & 0,006 \\
\hline Habilidades percibidas para la PBE & 0,11 & 0,055 & 0,18 & 0,003 \\
\hline Actitud hacia promoción de PBE & 0,31 & $<0,001$ & 0,32 & $<0,001$ \\
\hline Actitud de compañeros hacia PBE & 0,07 & 0,25 & 0,19 & 0,001 \\
\hline Nivel de conocimientos de inglés & 0,03 & 0,62 & 0,15 & 0,01 \\
\hline Nivel de conocimientos en informática & 0,30 & $<0,001$ & 0,42 & $<0,001$ \\
\hline Nivel de conocimientos en bioestadística & 0,31 & $<0,001$ & 0,38 & $<0,001$ \\
\hline Aspectos autovalorados & Conocimientos sobre PBE & Puntuación Global PBE \\
\hline & $\mathrm{R}$ & $\mathrm{p}$ & $\mathrm{R}$ & $\mathrm{p}$ \\
\hline Actitud percibida hacia PBE & 0,09 & 0,13 & 0,37 & $<0,001$ \\
\hline Conocimientos autovalorados sobre PBE & 0,44 & $<0,001$ & 0,26 & $<0,001$ \\
\hline Habilidades percibidas para la PBE & 0,43 & $<0,001$ & 0,30 & $<0,001$ \\
\hline Actitud hacia promoción de PBE & 0,17 & 0,003 & 0,36 & $<0,001$ \\
\hline Actitud de compañeros hacia PBE & 0,21 & $<0,001$ & 0,17 & 0,003 \\
\hline Nivel de conocimientos de inglés & 0,05 & 0,39 & 0,09 & 0,11 \\
\hline Nivel de conocimientos en informática & 0,20 & $<0,001$ & 0,40 & $<0,001$ \\
\hline Nivel de conocimientos en bioestadística & 0,17 & 0,003 & 0,40 & $<0,001$ \\
\hline$R=$ Rho Spearman $p$ : nivel de & & &
\end{tabular}

$R=$ Rho Spearman; $p$ : nivel de significación estadística

Fuente: Cuestionario de Competencias en Práctica Basada en la Evidencia (CACH-PBE), 2014.

\section{Discusión}

En el presente estudio se ha obtenido una versión del Cuestionario sobre Competencias en PBE de Estudiantes de Enfermería (CACH-PBE) adaptada culturalmente al contexto de Colombia y validada por expertos académicos colombianos.

Según los hallazgos encontrados, los estudiantes de enfermería de una Universidad de Cúcuta-Colombia, presentaron un nivel medio-alto en competencias para la $\mathrm{PBE}$, con una puntuación media de 3,58 sobre un máximo de 5. Es destacable considerar que la puntuación en la dimensión actitudes hacia la PBE es más alta que en habilidades y conocimientos de los estudiantes, lo que indica que existe una actitud favorable, aunque puede ser necesario un mayor énfasis en adquirir los conocimientos y las habilidades para aplicarlas.

En este aspecto, los resultados son similares a los obtenidos por Ruzafa et al. (21) en una muestra de estudiantes en una universidad en España. Por su parte Brooke et al. (27), en un estudio de corte cualitativo realizado en Inglaterra, evidenciaron que los estudiantes de enfermería, aunque percibieron la EBP y la investigación como de enormes proporciones y difícil de entender, las reconocieron como necesarias para su práctica actual y futura. Llasus et al. (28), en su estudio, mencionaron que la educación de enfermería debe centrarse en el aumento de la autoconfianza de los estudiantes durante la formación de sus competencias sobre PBE que apoyarán la participación en las conductas de ejecución de PBE. Así mismo, Tíscar (6) refirió que es indispensable que los profesionales de enfermería muestren una actitud que promueva la EBE, apoyando y favoreciendo la implantación de los cambios que de ella se derivan.

Un resultado inesperado fue la mayor puntuación global y en las habilidades que obtienen los hombres con respecto a las mujeres, aunque tanto en actitud como en conocimientos no se encontraron diferencias debidas al género. Este dato no había sido observado en estudio previos y, de confirmarse, podría indicar una mayor capacidad del grupo de estudiantes de enfermería varones para aplicar el modelo de PBE, aunque debe interpretarse con cautela.

La hipótesis inicial sobre el aumento en el nivel de 
competencias en PBE de los estudiantes, conforme avanzan en su formación académica, ha quedado confirmada con los resultados. En general, las puntuaciones en el cuestionario CACH-PBE, tanto global como en las 3 dimensiones consideradas, aumentan en los estudiantes de semestres superiores, con diferencias significativas en los estudiantes de años tercero y cuarto, respecto a los 2 primeros años.

Se observó una ligera disminución en las puntuaciones en los estudiantes del $2^{\circ}$ año con respecto al $1^{\circ}$, fenómeno para el cual no se encuentra explicación y podría deberse a fluctuación aleatoria. Igualmente, estos resultados coinciden con otros estudios similares $(18,21)$ que muestran mayores conocimientos y preparación para la $\mathrm{PBE}$ en estudiantes de cursos superiores.

De igual forma, se halló una asociación directa entre el nivel de competencias en PBE y un mayor número de horas de formación en metodología de investigación y en PBE recibidas por los estudiantes, tal como se estableció en la hipótesis inicial. Puesto que el número de horas formativas recibidas por los estudiantes va aumentando conforme avanzan en el desarrollo de los años de carrera, es posible interpretar que este grado de formación metodológica es responsable del aumento en las competencias en PBEy, sobre todo, en conocimientos y habilidades, en coincidencia con las conclusiones alcanzadas por Brown et al. (18) en estudiantes de dos universidades en EE.UU. Con base en estos resultados, es posible interpretar que el plan formativo del programa de enfermería, aunque no incluya ninguna materia específica sobre PBE, ofrece una formación en metodología que permite a los estudiantes avanzar en nivel de competencias sobre PBE.

Resulta interesante comparar los resultados del presente estudio con el de Ruzafa et al. (21) que sí incluía una asignatura específica sobre PBE desarrollada en el curso de tercer grado. Se pudo observar que, en la dimensión conocimientos, el aumento en la puntuación obtenida entre estudiantes de primer y cuarto año fue de 0,77 puntos en este estudio, pero fue más del doble (1,66 puntos) en el de Ruzafa et al. (21), con un importante aumento entre tercer y cuarto grado, que se atribuyó al efecto de esa asignatura específica. Sin embargo, el efecto de esa asignatura no es tan evidente en las otras dimensiones valoradas: actitudes y habilidades, en las que la magnitud de aumento entre primer y cuarto grado fue similar en ambos estudios.

Es de considerar que este proceso de adquisición de competencias a nivel de conocimientos y habilidades, en la actualidad, indiscutiblemente se está fortaleciendo con la oferta de diplomados, programas de maestría y doctorados hacia los profesionales de enfermería, los cuales ofrecen continuidad a las bases adquiridas en el pregrado y facilitan la adopción de la evidencia científica en su práctica, tal como se vio demostrado en el estudio de Pérez et al. (29) al identificar que, a mayor nivel académico y categoría profesional, se asocian mayores competencias sobre la PBE. Mariscal (30) menciona que con estos estudios se asumen nuevos roles que favorecen el cambio y el abandono de comportamientos rígidos que hoy por hoy se perciben en las áreas de cuidado.

Por otro lado, en el estudio se incluyeron 8 escalas de autovaloración de actitudes y conocimientos en diferentes aspectos de la PBE y metodológicos, con objeto de establecer en qué medida la autopercepción que tienen los estudiantes sobre sus competencias coincide con la medición que ofrece el cuestionario CACH-PBE. Se evidenció que la autovaloración de los estudiantes en actitudes y en conocimientos tiene alta correlación con las puntuaciones del cuestionario en estas dimensiones. Sin embargo, para la dimensión habilidades se observó que la mayor correlación ocurrió con la autovaloración de conocimientos en informática y en bioestadística, pero no en idioma inglés.

Estos resultados permiten interpretar que los estudiantes de la muestra analizada valoran como necesarios los conocimientos en manejo de ordenadores y en métodos estadísticos para obtener habilidades en la PBE. En cambio, la ausencia de correlación con el conocimiento de inglés indicaría que los estudiantes no consideran necesario obtener evidencias en este idioma y, por tanto, no realizan búsquedas en inglés como parte de las competencias en PBE.

Los anteriores hallazgos se pueden interpretar en el marco conceptual que realiza Ryan (15) en su revisión, al mencionar los factores que inciden en una actitud de los estudiantes más o menos favorable hacia la PBE. Entre los factores intrínsecos se encuentran: la intención de los estudiantes de aplicar el modelo de PBE, la confianza en sus capacidades para hacerlo 
ISSN-PRINT

1794-9831

E-ISSN 2322-7028

Vol. 14 No. 2

Jul - Dic 2017

Cúcuta, Colombia y la brecha percibida entre teoría y práctica. Como factores extrínsecos: el aprendizaje de la PBE dentro del contexto de las prácticas asistenciales y la implicación de los profesionales de enfermería que tutorizan a los estudiantes, la participación de los estudiantes en tareas de investigación o de búsqueda evidencias en colaboración con profesionales clínicos, la cultura institucional hacia la PBE y el nivel de formación en $\mathrm{PBE}$ de los profesionales asistenciales y de los docentes en las universidades, junto con la existencia de colaboración entre ambos.

Se pone de manifiesto que en la adquisición de competencias sobre la PBE por parte de los estudiantes de enfermería deben estar implicados no sólo los docentes académicos sino que también influyen los profesionales de enfermería que participan en la formación práctica de los estudiantes en los centros asistenciales.

\section{Conclusiones}

Los estudiantes del estudio presentaron una mediana de edad de 20 años, con predomino del género femenino, sin título académico previo y no desempeñaban ningún trabajo remunerado al momento del estudio. Con respecto a las características formativas, más de la mitad de ellos no tenía ninguna formación previa sobre PBE y solamente un pequeño porcentaje había recibido formación sobre el tema menor a 40 horas. Por su parte, cerca de la mitad de los participantes tenía algún nivel de formación en metodología de investigación.

Los estudiantes del programa de enfermería obtuvieron un nivel medio en competencias para la PBE, con una puntuación de 3,58 (sobre 5) en el cuestionario $\mathrm{CACH}-\mathrm{PBE}$ adaptado para Colombia. Estos estudiantes alcanzaron mayor puntuación en la dimensión actitud hacia la PBE que en las dimensiones habilidad y conocimiento. Se encontró que los estudiantes del género masculino evidenciaron mayor puntuación global y habilidad para la PBE.

La formación académica que reciben los estudiantes tiene un impacto positivo en la adquisición de competencias para la PBE, ya que los estudiantes de los últimos semestres obtuvieron mayor puntuación que los de primeros semestres. Posiblemente la formación metodológica incluida en las diferentes asignaturas del programa académico contribuye a este incremento en las competencias para la PBE, a pesar de que en el plan de estudios no existe ninguna asignatura específica sobre PBE.

De las ocho escalas de autovaloración, los resultados expresaron un predomino de niveles altos en la actitud hacia PBE y el nivel de conocimientos de informática, contra una menor apreciación para la actitud percibida de los compañeros hacia PBE, nivel de conocimientos de inglés y bioestadística, encontrándose una fuerte influencia de la competencia global en PBE con el nivel de conocimientos autodeclarado en bioestadística y en informática, así como también para la actitud percibida hacia la PBE.

La adquisición de competencias para la PBE en enfermería es un proceso complejo en el que estarían implicados los docentes académicos, pero también los profesionales de enfermería con quienes entran en contacto los estudiantes durante su aprendizaje práctico en los centros asistenciales.

\section{Conflicto de intereses}

Los autores declaran no tener ningún conflicto de intereses.

\section{Referencias bibliográficas}

1. Mulhall A. Nursing, research, and the evidence. Evid Based Nurs. 1998;1(1):4-6.

2. Eterovic-Díaz C, Stiepovich-Bertoni J. Enfermería basada en la evidencia y formación profesional. Cienc y enfermería [Internet]. 2010 Dic [cited 2016 Jul 16];16(3):9-14. Available from: http://www. scielo.cl/scielo.php?script=sci_arttext\&pid=S0717-95532010000300002\&lng=es\&nrm=iso\&tlng=es

3. Urra-Medina E, Retamal-Valenzuela C, Tapia-Pinto C, Rodríguez-Vidal M. Evidence based nursing: What is it, characteristics and dilemmas. Investig y Educ en Enfermería [Internet]. [cited 2016 Dec 15];28(1):108-18. Available from: http://www.scielo.org.co/scielo.php?script=sci arttext\&pid=S0120-53072010000100013\&lng=en\&nrm=iso\&tlng=es 
4. Luengo-Martínez C, Paravic-Klijn T. Autonomía Profesional. Factor clave para el ejercicio de la Enfermería Basada en la Evidencia. Index enferm. 2016;42-6.

5. DeBruyn RR, Ochoa-Marín SC, Semenic S. Barriers and Facilitators to Evidence-Based Nursing in Colombia: Perspectives of Nurse Educators, Nurse Researchers and Graduate Students. Investig y Educ en Enfermería [Internet]. [cited 2016 Jul 16];32(1):9-21. Available from: http://www.scielo.org. co/scielo.php?script=sci_arttext\&pid=S0120-53072014000100002\&lng=en\&nrm=iso\&tlng=en

6. González VT. Percepción de la práctica de enfermería basada en la evidencia de estudiantes de un master de investigación. ENE, Rev Enfermería. 2015;9(2).

7. Williams B, Perillo S, Brown T. What are the factors of organisational culture in health care settings that act as barriers to the implementation of evidence-based practice? A scoping review. Nurse Educ Today. 2015;35(2):e34-41.

8. Ansoain-Cilveti R. La evidencia científica en las unidades de enfermería como mejora de calidad. 2015;

9. González-Torrente S. Factores que influyen en la incorporación de la evidencia científica a la práctica diaria de las enfermeras de Atención Primaria. 2014.

10. de Pedro-Gómez J, Morales-Asencio JM, Abad AS, Veny MB, Vives GA, Campaner CP. Entorno de práctica de los profesionales de enfermería y competencia para la incorporación de la evidencia a las decisiones: situación en las Islas Baleares. Gac Sanit. 2011;25(3):191-7.

11. Sánchez-García I, López-Medina IM, Pancorbo-Hidalgo PL. Obstáculos percibidos por las enfermeras para la práctica basada en evidencias: Un estudio cualitativo. Enfermería clínica. 2013;23(6):279-83.

12. Ramírez-Vélez R, Domínguez MA, Morales-Osorio M, Meneses-Echavez JF, González-Ruiz K, Martínez-Torres J, et al. Estado actual de la investigación y principales barreras para la práctica basada en evidencia en fisioterapeutas colombianos. Fisioterapia. 2013;35(4):146-53.

13. Fawcett J. Tendencias de investigación en enfermería. Aquichán. 2014;14(3):289-93.

14. Raya DA. La investigación en enfermería en América Latina 2000. Rev Habanera Ciencias Médicas. 2011;10(3):396-409.

15. Ryan EJ. Undergraduate nursing students' attitudes and use of research and evidence-based practicean integrative literature review. J Clin Nurs. 2016;25(11-12):1548-56.

16. Upton P, Scurlock-Evans L, Upton D. Development of the Student Evidence-based Practice Questionnaire (S-EBPQ). Nurse Educ Today. 2016;37:38-44.

17. Ruzafa-Martinez M, Lopez-Iborra L, Moreno-Casbas T, Madrigal-Torres M. Development and validation of the competence in evidence based practice questionnaire (EBP-COQ) among nursing students. BMC Med Educ. 2013;13(1):1.

18. Brown CE, Kim SC, Stichler JF, Fields W. Predictors of knowledge, attitudes, use and future use of evidence-based practice among baccalaureate nursing students at two universities. Nurse Educ Today. 2010;30(6):521-7.

19. Spurlock D, Wonder AH. Validity and Reliability Evidence for a New Measure: The Evidence-Based Practice Knowledge Assessment in Nursing. J Nurs Educ. 2015;54(11):605-13.

20. Zelenikova R, Beach M, Ren D, Wolff E, Sherwood PR. Graduate nursing students' evaluation of EBP courses: A cross-sectional study. Nurse Educ Today. 2015;35(1):265-70.

21. Ruzafa-Martínez M, Molina-Salas Y, Ramos-Morcillo AJ. Competencia en práctica basada en la evidencia en estudiantes del Grado en Enfermería. Enfermería Clínica. 2016;26(3):158-64.

22. Falconí-Morales $C$, Brito Santacruz CJ, Verkovitch I. Integración de la enseñanza de la práctica de enfermería basada en la evidencia científica. Aquichan. 2015;15(4):541-53.

23. Pinto RC. Aplicación de la enfermería basada en la evidencia en los currículos de pregrado de enfermería en Santander, Colombia. Rev Cuid. 2015;6(1):900-5.

24. Escurra-Mayaute LM. Cuantificaoon de la validez de contenido por criterio de jueces. 1988; 6(12):103-111.

25. Aiken LR. Three coefficients for analyzing the reliability and validity of ratings. Educ Psychol Meas. $1985 ; 45(1): 131-42$. 
ISSN-PRINT

1794-9831

E-ISSN 2322-7028

Vol. 14 No. 2

Jul - Dic 2017

Cúcuta, Colombia

26. Republica de Colombia, Ministerio de Salud. Resolución N. 008430 del 4 de Octubre de 1993. Por la cual se establecen las normas científicas, técnicas y administrativas para la investigación en salud. El ministerio de Salud; 1993.

27. Brooke J, Hvalič-Touzery S, Skela-Savič B. Student nurse perceptions on evidence-based practice and research: An exploratory research study involving students from the University of Greenwich, England and the Faculty of Health Care Jesenice, Slovenia. Nurse Educ Today. 2015;35(7):e6-11.

28. Llasus L, Angosta AD, Clark M. Graduating baccalaureate students' evidence-based practice knowledge, readiness, and implementation. J Nurs Educ. 2014.

29. Pérez-Campos MA, Sánchez-García I, Pancorbo-Hidalgo PL. Knowledge, Attitude and Use of Evidence-Based Practice among nurses active on the Internet. Investig y Educ en Enfermería. 2014;32(3):451-60.

30. Crespo MIM. La Enfermería sin límites y los límites de la Enfermería. Enfermería clínica. 2012;22(3):115-7. 